\title{
A promoter of Fusarium graminearum Tri4 does not function when placed at the end of the trichothecene gene cluster
}

\author{
Takeshi TOKAI ${ }^{1}$, Yuichi NAKAJIMA ${ }^{2}$, Hinayo ICHIKAWA ${ }^{2}$, Kazuyuki MAEDA $^{2}$, Takumi NishiUCHI ${ }^{3}$, \\ Tetsuo KOBAYASHI ${ }^{2}$, Makoto KIMURA ${ }^{1,2, *}$
}

${ }^{1}$ Plant \& Microbial Metabolic Engineering Research Unit, DRI, RIKEN, 2-1 Hirosawa, Wako, Saitama 351-0198, Japan

${ }^{2}$ Department of Biological Mechanisms and Functions, Graduate School of Bioagricultural Sciences, Nagoya University, Furo-cho, Chikusa-ku, Nagoya, Aichi 464-8601, Japan

${ }^{3}$ Division of Functional Genomics, Advanced Science Research Center, Kanazawa University, 13-1 Takaramachi, Kanazawa, Ishikawa 920-0934, Japan

Key words : chromosomal location; Fusarium graminearum; gene cluster; GUS reporter; transcriptional activation

(Received January 14, 2013; Accepted January 29)

\begin{abstract}
The central region of the trichothecene gene cluster contains two pathway and two regulatory genes (four Tri genes) that are necessary for forming the trichothecene skeleton. One of these genes, Tri4, encodes a multifunctional cytochrome P 450 monooxygenase, and the expression of this gene is highly induced under trichothecene-inducing conditions. Apart from this gene cluster, Tri101 occurs as a single non-cluster gene, and the product of this gene contributes to the function of self-protection in Fusarium graminearum. The promoter activity of these Tri genes was compared with that of TEFl $\alpha$ (a highly expressed translation elongation factor 1-alpha) gene under toxin-inducing conditions by using the $\beta$-glucuronidase (GUS) gene as a reporter. The Tri101 promoter- and TEF1 $\alpha$ promoter-reporter fusions integrated at the end of the gene cluster showed activities that were similar to those of the original loci with respect to the timing and the level of GUS protein accumulation. However, as opposed to the normal pattern of Tri4 transcription from the original locus, no GUS activity was detected when the Tri4 promoter was placed at the end of the gene cluster. Thus, we can conclude that the position of the promoter in the gene cluster is important for native transcriptional regulation of Tri4 in trichothecene biosynthesis.
\end{abstract}

\section{Introduction}

Some plant pathogenic Fusarium species, such as the cereal pathogen F. graminearum, produce sesquiterpenoid mycotoxin trichothecenes that affect and thereby threatens the safety of cereal grains and related food products ${ }^{1)}$. Four genes located at the center of the trichothecene gene cluster, Tri4, Tri6, Tri5, and Tri10, are necessary for the formation of the basic trichothecene skeleton in the biosynthesis of Fusarium trichothecenes (Fig. 1). Tri5 encodes trichodiene synthase, which is necessary for the first cyclization step ${ }^{2}$, and Tri4 is

\footnotetext{
Corresponding Author

* Department of Biological Mechanisms and Functions, Graduate School of Bioagricultural Sciences, Nagoya University, Furo-cho, Chikusa-ku, Nagoya, Aichi 464-8601, Japan. Tel: + 81 52-789-5744. Fax: + 81 52-789-4087. E-mail: mkimura@ agr.nagoya-u.ac.jp
}

A full color PDF reprint of this article is available at the journal WEB site. 
a highly expressed multifunctional cytochrome P 450 monooxygenase gene required in the four subsequent oxygenation steps ${ }^{3,4)}$. The first trichohecene skeleton of Fusarium species, isotrichodermol, is biosynthesized as a result of the non-enzymatic second cyclization after the oxygenation steps. In addition to these trichothecene genes in the gene cluster, Tri101 occurs as a single non-clustered gene in the genome of $F$. graminearum $^{5}$. Tri101 expression is responsible for 3-O-acetylation of isotrichodermol, which is implicated in the self-protection of the fungus from the toxicity of trichothecenes.

Tri4 shares its promoter region with Tri6 (a $\mathrm{Cys}_{2} \mathrm{His}_{2}$ zinc finger transcription factor gene) ${ }^{6}$, whose activation is dependent on a functionally unidentifed regulatory protein encoded by Trilo (Fig. 1). Expression of these Tri genes is regulated in response to various environmental stimuli, including carbon source, nitrogen source, $\mathrm{pH}$, water activity, and oxidative stress ${ }^{7}$. When $F$. graminearum is grown under trichotheceneinducing conditions, Tri4 is induced and is expressed at a high level prior to the oneset of toxin production; expression of Tri101 then follows in accordance with the timing of toxin accumulation ${ }^{1)}$. We were thus interested in whether these promoters could be used as versatile tools for regulated expression of heterologous genes with respect to the expression patterns of the native Tri4 and Tri101 genes.

Promoter-reporter fusions are often used for comparing promoter activities, and must be integrated at the same locus to avoid chromosomal effects on transgene expression. In addition, the integration should not affect fungal growth or metabolism. With these criteria met, the activities of Tri4 and Tri101 promoters were evaluated using a promoter of the highly expressed $T E F 1 \alpha$ (translation elongation factor 1-alpha) gene ${ }^{8}$ as a reference for comparison.

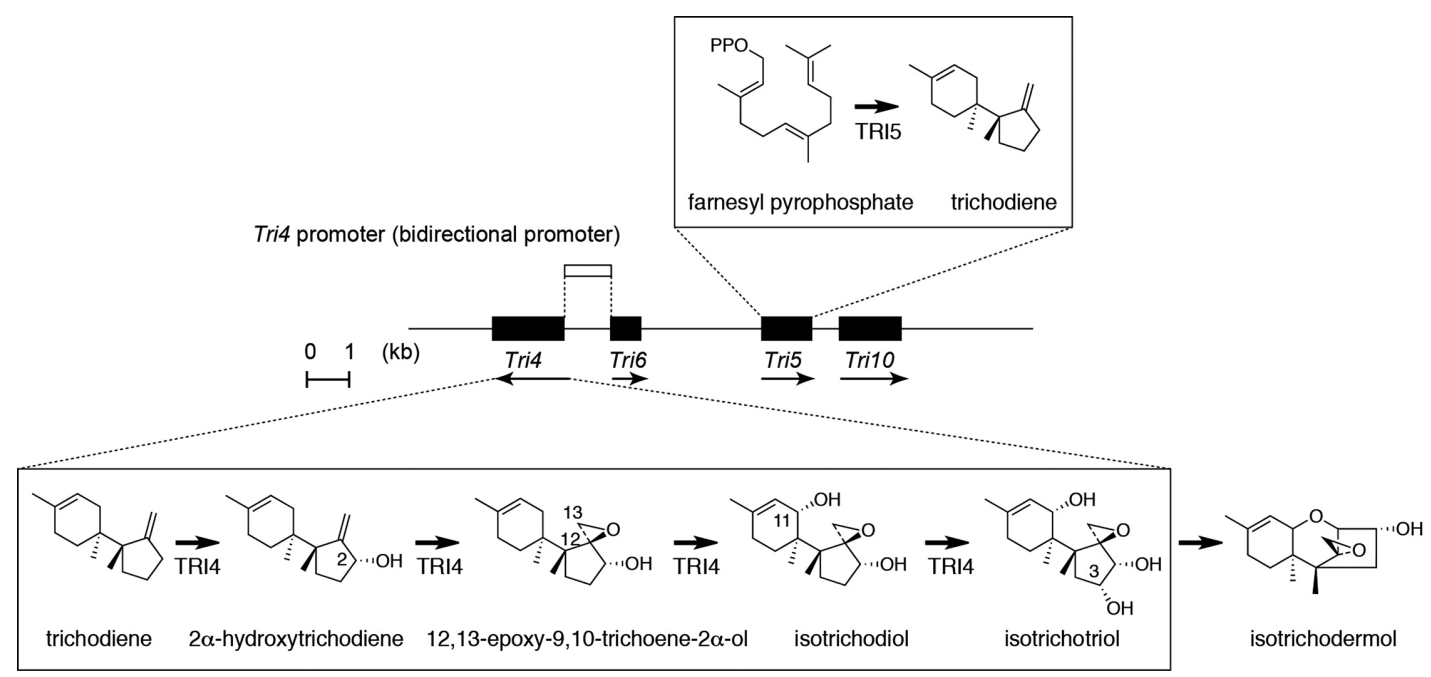

Fig. 1. The central region of the trichothecene gene cluster, comprising Tri4, Tri6, Tri5, and Tri10. The entire intergenic region $(1.1 \mathrm{~kb})$ between the start codons of Tri4 and Tri6 were used as a shared bidirectional promoter of Tri4 for the experiments. Enzymatic reactions catalyzed by TRI4 and TRI5 and described in the boxes. Note that these two pathway genes are sufficient to build the trichothecene skeleton because the second cyclization (isotrichotriol to isotrichodermol) proceeds spontaneously ${ }^{1)}$. 


\section{Materials and Methods}

\section{Fungal strains and culture conditions}

F. graminearum MAFF 111233, a strain that produces nivalenol (NIV)-type trichothecenes, was used as a host for the expression of a $\beta$-glucuronidase (GUS) gene $(\text { uidA })^{9)}$. For induction of trichothecene production, fungal strains were cultured in $100 \mathrm{ml}$ of modified rice flour (MRF) medium ${ }^{10)}$ (containing $4 \%$ rice (boiled for $30 \mathrm{~min}$ and glass filtered), $3 \%$ sucrose, and $0.1 \%$ yeast extract) on a gyratory shaker $(160 \mathrm{rpm})$ at $22{ }^{\circ} \mathrm{C}$. Strain MAFF 111233 mainly produces 4,15-diacetylnivalenol (4,15-diANIV) in MRF medium.

Amplification of promoters used for the assay $\quad$ Tri4 and Tri101 promoters were amplified from the genomic DNA of $F$. graminearum by performing PCR using KOD Neo (ToYoBo Life Science) with primers MFTri 4 Pro-U2 (5'-AAAAAGATGGCTTGATGGGAGGGG-3') and MFTri 4 Pro-D (5'CTTTTCAAAGTTCAAGTCTTTGATAG-3') (with an amplicon size of $1.1 \mathrm{~kb}$ ), and primers MFTri101ProU ( 5'-AATGGATGAGTCAATCGATACGCA-3') and MFTri 101 Pro-D ( 5'-TTTGGTGGTGTTTGTAAATCACTT- 3') (amplicon size $0.7 \mathrm{~kb}$ ), respectively. The promoter of TEF1 $\alpha$ (AN4218.3) was amplified by perfoming PCR of the genomic DNA of Aspergillus nidulans NBRC 33017 with primers AN4218TEFPro-U3 (5' - TAGCGACTTAGAGTGCACGCTCAAAAGA-3') and AN4218TEFPro-D (5'-GGTGAAGGTTGTGTTATGTTTTGT-3’) (amplicon size $1.1 \mathrm{~kb}$ ).

Construction of promoter-GUS fusion vectors for integration into the Tri14 locus $\quad \mathrm{pGR}-\mathrm{Srf}-\mathrm{hph}$ (Fig. 2A), a derivative of pHF312 (Tokai et al. unpublished; constructed from pBF312 ${ }^{11)}$ by replacing $b s d$, a blasticidin S deaminase gene, with $h p h$, a hygromycin phosphotransferase gene), is a promoter-cloning GUS vector that contains $h p h$ for selection of fungal transformants and a $\operatorname{Srf}$ I site for insertion of promoters amplified by PCR. For targeted integration of this vector into the Tri $14^{12)}$ locus, we constructed pGR-Srf-hph-MFTri14 by replacing the short NotI-SpeI linker fragment of pGR-Srf-hph with a NotI-SpeI digested inverse PCR (IPCR) product containing each side of the Tril4 region. This IPCR product was generated in the following manner: (1) the Tri14 region was amplified by perfoming PCR with NheI site-containing primers DLTri14-U/Nhe (5' -AAGCTAGCGTATCAGTCTCCAATCGGTC- 3’) and DLTri 14 -D/Nhe ( 5'-CGGCTAGCGTTGACGAACATTGCTGACC-3'), (2) the amplified product was digested using NheI and then was self-ligated, and (3) this self-ligated DNA was used as a template for performing IPCR with primers INVTri 14 -D/Not ( 5' -ACAGCGGCCGCGTATAGGTTGCTTCAAG- 3') and INVTri 14 -U/Spe ( 5'-GAACTAGTGTTGTGATCAAGCAGACTCC- 3') containing Not I and SpeI sites, respectively. The promoter fragments amplified by performing PCR were cloned into the Srf I-cloning site of pGR-Srf-hph-MFTri 14 (yielding vectors $\mathrm{pP}_{\text {Tri4 }}$ GR-hph-MFTri14, $\mathrm{pP}_{\text {Tri10I- }}$ GR-hph-MFTri14, and $\mathrm{P}_{\text {TEFIa }}$ GR-hph-MFTri14), linearized with NheI, and were used to transform $F$. graminearum according to the method described previously ${ }^{4}$.

Screening of transformants Transformants with homologous recombination were screened by performing long PCR (LA-PCR) using LA-Taq (Takara Bio Inc.). Primers FgTri 14 -LAU (5'-TAGCTGGAATGTCGCCAAAAAGACTCTG-3') and FgTri 14 -LAD (5'-CCTTACTTTAGCTTCAACGACCGAACAC-3') were used for amplifying the Tril4 region (Fig. 2B). 
(A)

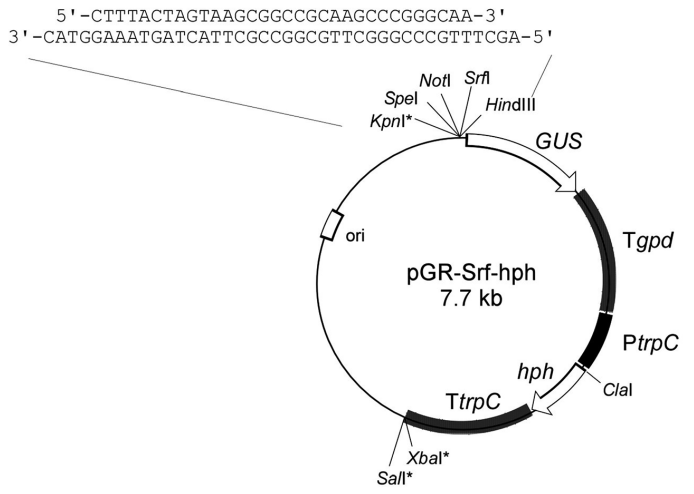

(B)

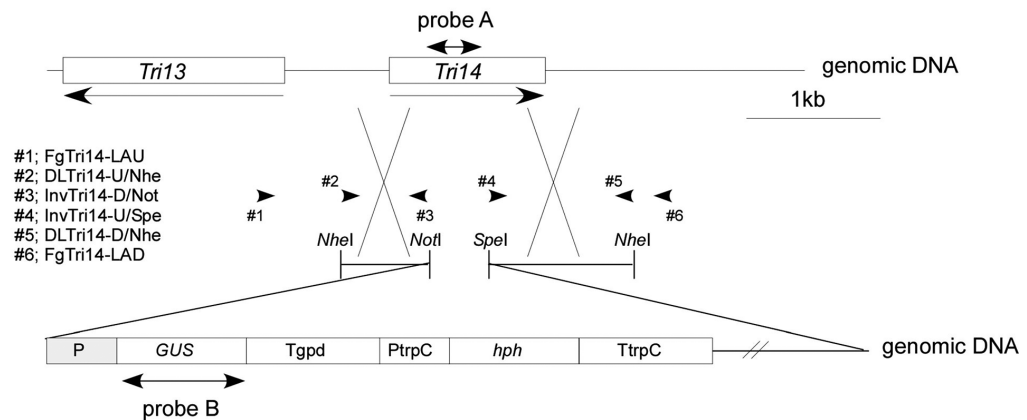

(C)

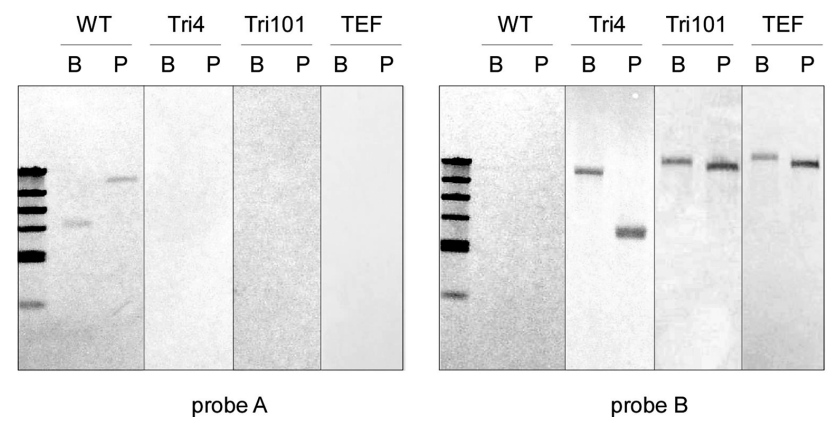

Fig. 2. Generation of $F$. graminearum transformants for evaluating promoter activities. A. Structure of pGR-Srf-hph used for constructing pGR-Srf-hph-MFTri 14, a promoter-reporter fusion vector designed for targeted integration at the Tril4 locus. A KpnI-HindIII fragment of the A. nidulans trpC promoter in pGR $101^{5)}$ was replaced with a synthetic linker containing KpnI, SpeI, NotI, Srf I, and HindIII sites (shown in the figure). After SacI digestion and blunting, the larger vector fragment was ligated to a blunted $S p h$ I fragment of pHF312 containing the Cryphonectria parasitica gpd terminator (Tgpd), the $A$. nidulans trpC promoter, $h p h$, and the $A$. nidulans trpC terminator. B. Diagram showing targeted integration of the promoter-GUS fusion vectors to the Tri14 locus. Promoters of F. graminearum Tri4, Tri101, and A.

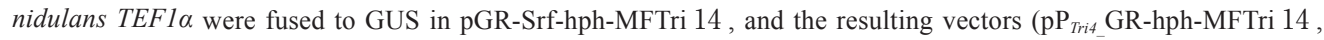
$\mathrm{pP}_{\text {TriloI_- }}$ GR-hph-MFTri14, and $\mathrm{P}_{\text {TEFla_- }}$ GR-hph-MFTri14) were targeted to the Tri14 locus. Arrow heads indicate annealing sites of primers used for screening of the transformants (\#1 and \#6) and for constructing pGR-Srf-hph-MFTri14 (\#2\#5). Probes used for Southern blot analyses are indicated by double arrows. C. Southern blot analyses of wild-type (WT) and transformants. The result of a representative transformant is shown. DNA was digested using BamHI (B) or PstI (P),

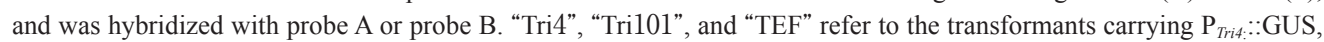

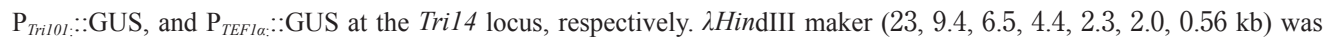
loaded to the left of the WT lanes. 
Nucleic acid hybridizations DNA probes for Southern and northern blot analyses were generated by labeling fragments with digoxigenin using the PCR DIG probe synthesis kit (Roche Applied Science). For Southern blot analyses, probe A was obtained by performing PCR of genomic DNA with primers Fgtri14 probe-U (5'-TTGAATTCCCAGGTCTCAGTCACG- $3^{\prime}$ ) and Fgtri 14 probe-D (5'-CCAATGATGTTGTCGGGAACAATC-3'); probe B was amplified from pGR-Srf-hph by using primers GUS-ATG (5'-ATGTTACGTCCTGTAGAAACCCCA-3') and GUS-TGA (5'-TTCATTGTTTGCCTCCCTGCTGCG-3'). For northern blot analyses, a Tri4 probe was amplified from cDNA with primers GzTri4/TAA and GzTri4/ATG/SacI); a Tri101 probe was amplified with primers U101 and D101 ${ }^{5}$. Standard hybridization techniques recommended by the manufacturer were used for Southern and northern blot analyses.

In vitro GUS assay One gram of mycelia was ground in a mortar in liquid nitrogen and suspended in 2 $\mathrm{ml}$ of GUS extraction buffer ( $50 \mathrm{mM}$ phosphate buffer, $\mathrm{pH} 7.0$, containing $10 \mathrm{mM} \beta$-mercaptoethanol, $10 \mathrm{mM}$ EDTA, $0.1 \% N$-lauroylsarcosine sodium salt, $0.1 \%$ Triton X-100). The mycelial suspension was centrifuged at $4{ }^{\circ} \mathrm{C}$ for $15 \mathrm{~min}$ at $20,400 \times \mathrm{g}$. The supernatant was filtered through a Millex syringe filter unit $(0.45 \mu \mathrm{m}$, Millipore) and used to evaluate the promoter activity by performing GUS assay. The GUS assay was performed using a $\beta$-glucuronidase (GUS) Reporter Gene Activity Detection kit (MGT Inc.), as per the manufacturer's instructions. Briefly, $10 \mu 1$ of crude enzyme was mixed with $90 \mu 1$ of GUS Assay Buffer supplied in the kit (final $0.09 \mathrm{mM} 4$-methylumbelliferyl $\beta$-D-glucuronide), incubated for $30 \mathrm{~min}$ at $37^{\circ} \mathrm{C}$, and then the reaction was terminated by adding $200 \mu 1$ of Carbonate Stop buffer (included in the kit). The concentration of 7-hydroxy-4-methylcoumarin (4-MU) formed was determined by measuring the fluorescence of 4 -MU with a Wallac ARVO SX 1420 MultiLabel Counter (Perkin Elmer) using excitation and emission wavelengths of 365 and $469 \mathrm{~nm}$, respectively. The GUS activity was calculated in pmol of 4-MU formed per min per $\mu$ g of protein.

Chemical and biochemical analyses Trichothecenes were extracted using ethyl acetate, developed on a TLC plate (Merck $\mathrm{F}_{254}$ silica TLC) using ethyl acetate/toluene $(3: 1)$ as a solvent, and visualized with a chromogenic reagent, as per a previously described method ${ }^{13)}$. Protein concentrations were determined using a Pierce BCA TM Protein assay kit (Thermo Scientific).

\section{Results and Discussion}

A $\beta$-glucuronidase (GUS) gene was introduced downstream of each promoter and was used as a reporter to evaluate the promoter activity. A portion of the coding region of Tri $14^{12}$, which is located at the end of the trichothecene gene cluster, was chosen as the integration site for the promoter-GUS fusions; this gene is not necessary for the trichothecene biosynthesis steps, and the deletion mutant normally produces trichothecenes when the fungus is grown under toxin-inducing conditions. Promoter fragments amplified by PCR were cloned into the Srfl site of pGR-Srf-hph-MFTri 14, and the sequence was verified by sequencing. After digestion with NheI, the vectors were transformed to F. graminearum.

The genomic DNA of the transformants were screened for the occurrence of the promoter-GUS fusions at the Tri14 locus (Fig. 2 B). Strains with double crossing-over homologous recombination gave LA-PCR products that were approximately $10 \mathrm{~kb}$ in length; primers FgTri14-LAU and FgTri14-LAD (see Materials 
and Methods), whose annealing sites are separated by $3 \mathrm{~kb}$ in the wild-type genome (Fig. 2B) had been used. Figure $2 \mathrm{C}$ shows representative Southern blot data of such transformants carrying $\mathrm{P}_{T r i 4}:$ GUS, $\mathrm{P}_{T r i 101}:$ GUS, and $\mathrm{P}_{\text {TEFIa: }}:$ GUS, further confirming the integration of the promoter-GUS fusions at the Trilt locus. When hybridized with probe A (a deleted region of Tril4 containing neither the BamHI nor the PstI site), single bands detected on the DNA blots of the wild-type (a $5 \mathrm{~kb}$ Bam $\mathrm{HI}$ fragment and a Pst Iragment larger than 10 $\mathrm{kb}$ ) were lost from those of the transformants carrying $\mathrm{P}_{\text {Tri }}:: \mathrm{GUS}, \mathrm{P}_{\text {Trilo1 } 1}:$ GUS, and $\mathrm{P}_{\text {TEFI } 1:}:$ GUS at the Tril4 locus (Fig. $2 \mathrm{C}$; left panel). In contrast, single bands were detected on the blots of these transformants with probe B (a coding region of GUS containing neither the BamHI nor the PstI site), and these bands were absent in the case of the wild-type (Fig. 2C; right panel).

The GUS activity of these transformants were measured at various time points after transfer to the trichothecene-inducing MRF medium. The wet weight of these transformants showed less than $20 \%$ of variability (compared to that of the $\mathrm{P}_{\text {TEFIa: }}:$ GUS transformant) at each time point (data not shown). The timing and level of trichothecene accumulation was not significantly altered by the disruption of Tri14 (Fig. 3 A). Constitutively, high levels of GUS activitiy was observed in the mycelia of the $\mathrm{P}_{\text {TEFIL }:}:$ GUS transformant; the activity was highest at $24 \mathrm{~h}$, and then it gradually decreased at 48,72 , and $96 \mathrm{~h}$ (Fig. $3 \mathrm{~B}$ ). This pattern of expression is reasonable because the promoters of housekeeping genes are transcribed during active growth, and their expression declines after the transition of the organism to the stationay phase. In contrast, the $\mathrm{P}_{\text {Trilol }}:$ GUS transformant showed a GUS expression pattern consistent with the acumulation of trichothecenes under trichothecene-inducing culture conditions. However, integration of the $\mathrm{P}_{T r i 4}:$ GUS fusion vector $\left(\mathrm{pP}_{T r i 4}\right.$ GR-hph-MFTri14) into the Tri14 locus resulted in undetectable levels of GUS activity at all time points. This lack of detectable GUS activity cannot be attributed to an unexpected mutation that may have been accidentally acquired during transformation because other $\mathrm{pP}_{\text {Tri4 }}$ GR-hph-MFTri 14 transformants carrying the promoter-reporter fusion at the Tril4 locus (and also those carrying it at ectopic sites) also failed to produce the GUS protein (data not shown). Data obtained from the northern blot analyses in the same time course experiment confirmed a normal transcript accumulation pattern of Tri4 like that in the native promotors ${ }^{14)}$, which was also observed for Tri101 (Fig. 3C). These results suggest that the Tri4 promoter does not support sufficient transcription levels of heterologous genes when integrated at non-native sites.

The $1.1 \mathrm{~kb}$ intergenic region of divergently transcribed Tri4 and Tri6 constitutes a shared bidirectional promoter. In strain MAFF 111233 used in this study, the Tri4 promoter region contains two closely positioned putative TRI6-binding sites, $\mathrm{T}_{4} \mathrm{~A}_{\mathrm{Fg}}$ ( $\underline{\mathrm{TCAGGCCT}}$ ) and $\mathrm{T}^{4} \mathrm{~B}_{\mathrm{Fg}}(\underline{\mathrm{CC}} \underline{\mathrm{AGGCCT}})$, between bp -377 and -361 with respect to the ATG start codon of Tri4 (underlined nuclotides indicate matches to consensus sequences ${ }^{15}$, TNAGGCCT, and/or the minimum sequence required for TRI 6 binding YNAGGCC $\left.{ }^{15}\right)$ ). These putative

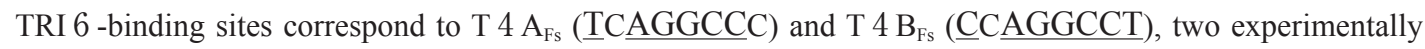
dertermined TRI 6 -binding sites in the Tri4 promoter of Fusarium sporotrichioides, with regard to their positions from the ATG start codon ${ }^{15}$. Therefore, the affinity of the TRI6 protein to the F. graminearum Tri4 promoter is comparable and cannot be the cause of undetectable levels of GUS expression from the Tril4 locus.

Tri4 is situated $17.5 \mathrm{~kb}$ upstream of Tril 4 that demarcates the end of the gene cluster. The inability of the Tri4 promoter to cause heterologous gene expression from a non-native locus may suggest the necessity of additional elements located in the coding region of Tri4 for transcriptional activation of its own gene. Alternatively, the physical linkage of Tri genes or chromatin remodeling at the central region of the gene cluster may 
(A)

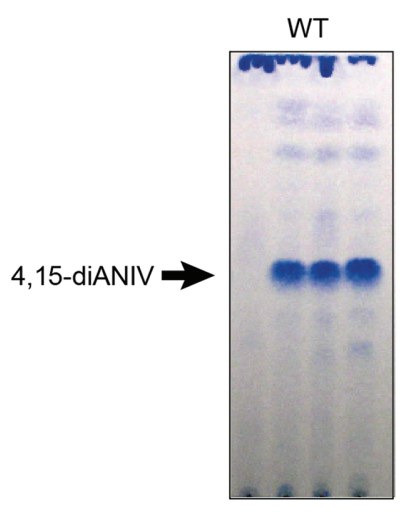

24487296

(B)

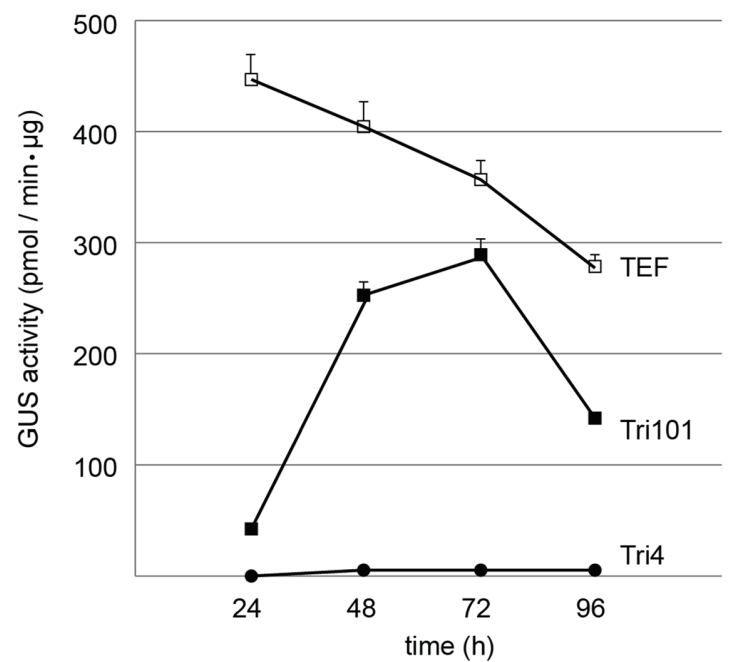

Tri101

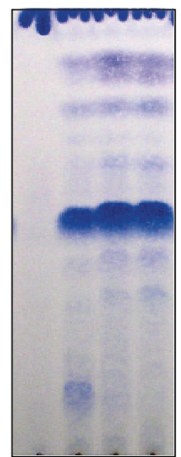

24487296

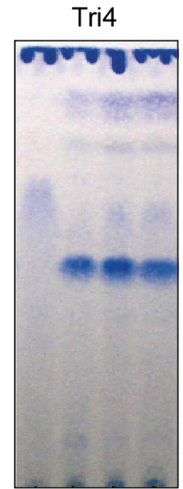

24487296

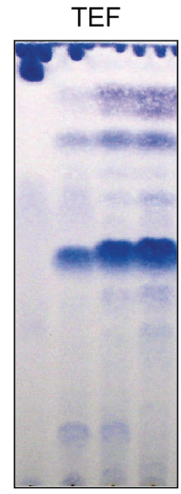

24487296 (h)

(C)

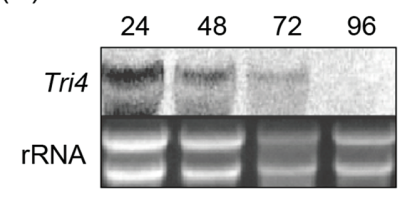

(h)

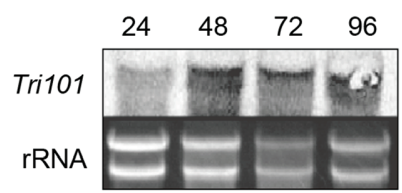

(h)

Fig. 3. The Tri4 promoter does not work when integrated at the Tri14 locus. In A and B, "Tri4", "Tri101", and "TEF" refer to the same transformants as described in Fig. 2 C. A. TLC analysis of trichothecenes extracted from the culture of the wild-type (WT) and the transformants at each time point of the GUS assay. Strain MAFF 111233 produces 4,15-diANIV in liquid culture. Ethyl acetate extract from $5 \mathrm{ml}$ of the culture was loaded in each lane. In consideration of the fact that the cultures are derived from independent strains, the data indicate that their trichothecene levels do not significantly differ each other. B. GUS activities of the transformants at 24, 48, 72, and $96 \mathrm{~h}$ after transfer to MRF medium. C. Northern blot analyses of Tri genes. Expressions of Tri4 and Tri101 in the transformants were monitored at each time point of the GUS assay.

have a regulatory role in the epigenetic activation of Tri4 and some other Tri genes. In either case, the distance between the native Tri4 locus and the $\mathrm{P}_{T r i 4}:$ :GUS reporter fusion is too long to exert regional control over the transcriptional activity of the Tri14 locus. Similar position-dependent regulation of clustered secondary metabolite (SM) gene expression has also been demonstrated for the ver $-1^{16)}$ and $n o r-1^{17)}$ genes in aflatoxin biosynthesis; GUS expression from their promoters that were engineered to reside outside of the aflatoxin gene cluster was marginal to absent. These results led to the conclusion that positional effects are important in the expression of aflatoxin biosynthetic genes ${ }^{18)}$.

In conclusion, we found that a gene located in the centeral region of the trichothecene gene cluster is 
subject to position-dependent regulation and that the promoter does not function when placed at the the end of the gene cluster. Future studies designed to determine factors (other than pathway-specific transcription factors) necessary for the complete activation of the promoter may lead to a broader understanding of the significance of gene clustering and the regulatory mechanisms of clustered SM genes.

\section{Acknowledgement}

This work was supported in part by JSPS KAKENHI Grant Number 23658084.

\section{References}

1) Kimura, M., Tokai, T., Takahashi-Ando, N., Ohsato, S., Fujimura, M.: Molecular and genetic studies of Fusarium trichothecene biosynthesis: pathways, genes, and evolution. Biosci Biotechnol Biochem, 71, 2105-2123 (2007)

2) Hohn, T. M., Beremand, P. D.: Isolation and nucleotide sequence of a sesquiterpene cyclase gene from the trichothecene-producing fungus Fusarium sporotrichioides. Gene, 79, 131-138 (1989)

3) Hohn, T. M., Desjardins, A. E., McCormick, S. P.: The Tri4 gene of Fusarium sporotrichioides encodes a cytochrome P450 monooxygenase involved in trichothecene biosynthesis. Mol Gen Genet, 248, 95-102 (1995)

4) Tokai, T., Koshino, H., Takahashi-Ando, N., Sato, M., Fujimura, M., Kimura, M.: Fusarium Tri4 encodes a key multifunctional cytochrome P450 monooxygenase for four consecutive oxygenation steps in trichothecene biosynthesis. Biochem Biophys Res Commun, 353, 412-417 (2007)

5) Kimura, M., Kaneko, I., Komiyama, M., Takatsuki, A., Koshino, H., Yoneyama, K., Yamaguchi, I.: Trichothecene $3-O$-acetyltransferase protects both the producing organism and transformed yeast from related mycotoxins. Cloning and characterization of Tri101. J Biol Chem, 273, 1654-1661 (1998)

6) Proctor, R. H., Hohn, T. M., McCormick, S. P., Desjardins, A. E.: Tri6 encodes an unusual zinc finger protein involved in regulation of trichothecene biosynthesis in Fusarium sporotrichioides. Appl Environ Microbiol, 61, 1923-1930 (1995)

7) Merhej, J., Richard-Forget, F., Barreau, C.: Regulation of trichothecene biosynthesis in Fusarium: recent advances and new insights. Appl Microbiol Biotechnol, 91, 519-528 (2011)

8) Kitamoto, N., Matsui, J., Kawai, Y., Kato, A., Yoshino, S., Ohmiya, K., Tsukagoshi, N.: Utilization of the TEF1-alpha gene (TEF1) promoter for expression of polygalacturonase genes, pgaA and pgaB, in Aspergillus oryzae. Appl Microbiol Biotechnol, 50, 85-92 (1998)

9) Jefferson, R. A.: The GUS reporter gene system. Nature 342, 837-838 (1989)

10) Ochiai, N., Tokai, T., Takahashi-Ando, N., Fujimura, M., Kimura, M.: Genetically engineered Fusarium as a tool to evaluate the effects of environmental factors on initiation of trichothecene biosynthesis. FEMS Microbiol Lett, 275, 53-61 (2007)

11) Banno, S., Kimura, M., Tokai, T., Kasahara, S., Higa-Nishiyama, A., Takahashi-Ando, N., Hamamoto, H., Fujimura, M., Staskawicz, B. J., Yamaguchi, I.: Cloning and characterization of genes specifically expressed during infection stages in the rice blast fungus. FEMS Microbiol Lett, 222, 221-227 (2003)

12) Brown, D. W., McCormick, S. P., Alexander, N. J., Proctor, R. H., Desjardins, A. E.: Inactivation of a 
cytochrome P 450 is a determinant of trichothecene diversity in Fusarium species. Fungal Genet Biol, 36, 224-233 (2002)

13) Takitani, S., Asabe, Y., Kato, T., Suzuki, M., Ueno, Y.: Spectrodensitometric determination of trichothecene mycotoxins with 4-( $p$-nitrobenzyl)pyridine on silica gel thin-layer chromatograms. J Chromatogr, 172, 335-342 (1979)

14) Ponts, N., Pinson-Gadais, L., Barreau, C., Richard-Forget, F., Ouellet, T.: Exogenous $\mathrm{H}_{2} \mathrm{O}_{2}$ and catalase treatments interfere with Tri genes expression in liquid cultures of Fusarium graminearum. FEBS Lett, 581, 443-447 (2007)

15) Hohn, T. M., Krishna, R., Proctor, R. H.: Characterization of a transcriptional activator controlling trichothecene toxin biosynthesis. Fungal Genet Biol, 26, 224-235 (1999)

16) Liang, S. H., Wu, T. S., Lee, R., Chu, F. S., Linz, J. E.: Analysis of mechanisms regulating expression of the ver-1 gene, involved in aflatoxin biosynthesis. Appl Environ Microbiol, 63, 1058-1065 (1997)

17) Chiou, C. H., Miller, M., Wilson, D. L., Trail, F., Linz, J. E.: Chromosomal location plays a role in regulation of aflatoxin gene expression in Aspergillus parasiticus. Appl Environ Microbiol, 68, 306-315 (2002)

18) Palmer, J. M., Keller, N. P.: Secondary metabolism in fungi: does chromosomal location matter? Curr Opin Microbiol, 13, 431-436 (2010) 